\title{
QCD Corrections to Higgs-Boson Production at Proton-Proton Colliders
}

\author{
D. Graudenz \\ Lawrence Berkeley Laboratory, University of California, Berkeley, California 94720(a) \\ and Institut für Theoretische Physik, Rheinische-Westfalische Technische Hochschule Aachen, \\ D-5100 Aachen, Federal Republic of Germany \\ M. Spira \\ Institut für Theoretische Physik, Rheinisch-Westfälische Technische Hochschule Aachen, \\ D-5100 Aachen, Federal Republic of Germany \\ and Deutsches Elektronen-Synchrotron (DESY), D-2000 Hamburg 52, Federal Republic of Germany ${ }^{(a)}$ \\ P. M. Zerwas \\ Deutsches Elektronen-Synchrotron (DESY), D-2000 Hamburg 52, Federal Republic of Germany
}

(Received 30 November 1992)

\begin{abstract}
Gluon fusion is the main production mechanism for Higgs bosons with masses up to several hundred $\mathrm{GeV}$ in $p p$ collisions at the CERN Large Hadron Collider and Superconducting Super Collider. We present the QCD corrections to the fusion cross section for arbitrary Higgs boson and top quark mass values: $g g \rightarrow H(g), g q \rightarrow H q$, and $q \bar{q} \rightarrow H g$. The QCD corrections are positive and they increase the cross section $\sigma(p p \rightarrow H)$ by about a factor of 1.5 to 1.7. The analysis applies to the production of Higgs particles in the standard model and also to the production of the $C P$-even Higgs particles in extensions of the Higgs sector as required, for example, by supersymmetric theories.
\end{abstract}

PACS numbers: 12.38.Bx, 13.85.Qk, 14.80.Gt

Most of the building blocks of the standard model have been tested to a very high precision in recent years. However, the Higgs mechanism [1], one of the cornerstones of this theory, has not yet been proven experimentally to be the basic mechanism for the generation of the fundamental particle masses. To accommodate the well-established electromagnetic and weak phenomena, this mechanism requires the existence of at least one weak isodoublet scalar field of which 1 degree of freedom manifests itself as a real physical particle for small enough masses. The discovery of the Higgs particle is the experimentum crucis for the canonical formulation of the electroweak interactions.

Theoretical consistency restricts the mass of the Higgs boson in the standard model to less than about $700 \mathrm{GeV}$ [2]. Higher mass values may nevertheless be realized in extensions of the (canonical) standard model. A lower limit of about $60 \mathrm{GeV}$ has been set by the CERN $e^{+} e^{-}$ Collider (LEP) experiments [3]. Depending on the maximum energy LEP will reach, the search for Higgs particles can eventually be extended up to masses of about $90 \mathrm{GeV}$. To continue the search beyond this limit, new accelerators are needed. While $e^{+} e^{-}$colliders with a c.m. energy of $500 \mathrm{GeV}$ are ideal machines to investigate Higgs particles in the intermediate mass range below the $Z Z$ decay threshold, the multi-TeV $p p$ CERN Large Hadron Collider (LHC) and Superconducting Super Collider (SSC) can sweep the mass range up to the theoretical upper limit [4].

For Higgs boson masses up to $\sim 700 \mathrm{GeV}$ the dominant production mechanism in high-energy proton-proton col- lisions is gluon-gluon fusion [5]. The Higgs bosons couple to gluons through a heavy-quark triangle loop. In this Letter we present the QCD corrections to the cross section $\sigma(p p \rightarrow H+X)$ of the fusion process,

$$
g g \rightarrow H(g) \text { and } g q \rightarrow H q, \quad q \bar{q} \rightarrow H g
$$

for arbitrary Higgs boson and (loop) quark masses, Fig.1. This analysis extends previous work of Refs. $[6,7]$ in which the Higgs boson mass was assumed to be much smaller than the loop-quark mass. The analysis of the next-to-leading-order QCD corrections to the Higgs production cross section is required for two reasons: (i) The lowest order prediction of the cross section $\sigma(p p \rightarrow H)$ depends strongly on the renormalization and factorization scales, in particular, for heavy Higgs boson masses. The next-to-leading order reduces this scale dependence of the cross section significantly. (ii) Since the QCD corrections turn out to be positive and large, the calculation is a posteriori also of high experimental significance. The transverse momentum spectra of the Higgs bosons have been considered at various levels of theoretical refinement in Refs. [8, 9].

Heavy quarks $Q$ provide the dominant contribution to the coupling of Higgs bosons to gluons. Neglecting finitewidth effects, the cross section for the production of Higgs particles in $p p$ collisions is given to lowest order (LO) [Fig.1(a)] by [5-7]

$$
\sigma_{\mathrm{LO}}(p p \rightarrow H+X)=\sigma_{0} \tau_{H} \frac{d \mathcal{L}^{g g}}{d \tau_{H}}
$$

with 


$$
\begin{gathered}
\sigma_{0}=\frac{G_{F} \alpha_{s}^{2}}{288 \sqrt{2} \pi}\left|\sum_{Q} \frac{3}{2} \tau_{Q}^{-1}\left[1+\left(1-\tau_{Q}^{-1}\right) f\left(\tau_{Q}\right)\right]\right|^{2}, \\
f\left(\tau_{Q}\right)= \begin{cases}\arcsin { }^{2} \sqrt{\tau_{Q}} & \tau_{Q}<1, \\
-\frac{1}{4}\left[\ln \frac{1+\sqrt{1-\tau_{Q}^{-1}}}{1-\sqrt{1-\tau_{Q}^{-1}}}-i \pi\right]^{2}, & \tau_{Q}>1,\end{cases}
\end{gathered}
$$

and $d \mathcal{L}^{g g} / d \tau_{H}$ denoting the gluon luminosity. The scaling variables are as usual defined by $\tau_{Q}=m_{H}^{2} / 4 m_{Q}^{2}$ and $\tau_{H}=m_{H}^{2} / s$, where $\sqrt{s}$ is the total c.m. energy of the proton collider.

The QCD corrections to the lowest order diagram [Fig. 1(a)] consist of gluon-quark and Higgs-boson-quark vertex corrections, propagator corrections, and the associated counter terms. An example is shown in Fig. 1(b). The renormalization program has been carried out in the $\overline{\mathrm{MS}}$ (modified minimal subtraction) scheme. The mass $m_{Q}$ of the heavy quark is defined at the pole of the quark propagator. The renormalization of the Higgsboson-quark vertex is connected with the renormalization of the quark mass and the quark wave function, $Z_{H Q Q}=Z_{Q}-\delta m_{Q} / m_{Q}[10]$. In addition to these virtual corrections, the gluon radiation off the initial state gluons and off the heavy-quark loop must be taken into account; Fig. 1(c). After adding up these corrections, ultraviolet and infrared singularities cancel. Leftover collinear singularities are absorbed into the renormalized parton densities [11] which we define in the $\overline{\mathrm{MS}}$ scheme. Finally the subprocesses $g q \rightarrow H q$ and $q \bar{q} \rightarrow H g$ must be added [Fig. 1(d)].

The result for the cross section can be cast into the following form:

$$
\begin{aligned}
\sigma(p p \rightarrow H+X)= & \sigma_{0}\left[1+C\left(\tau_{Q}\right) \frac{\alpha_{s}}{\pi}\right] \tau_{H} \frac{d \mathcal{L}^{g g}}{d \tau_{H}} \\
& +\triangle \sigma_{g g}+\triangle \sigma_{g q}+\triangle \sigma_{q \bar{q}} .
\end{aligned}
$$

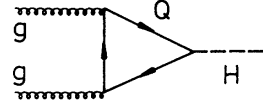

(a)

(c1)

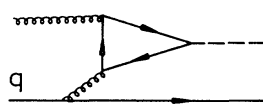

(d1)

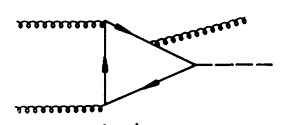

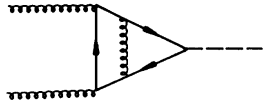

(b)

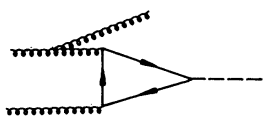

(c2)

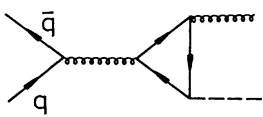

(d2)
FIG. 1. Generic diagrams for the production of Higgs bosons in gluon-gluon, gluon-quark, and quark-antiquark collisions: (a) lowest order $g g$ contribution; (b) vertex corrections; (c) real gluon radiation; (d) Higgs production in $g q$ and $q \bar{q}$ collisions.

The coefficient $C\left(\tau_{Q}\right)$ denotes the contribution from the virtual two-loop corrections regularized by the infrared singular part of the cross section for real gluon emission. This coefficient splits into the infrared term $\left(\pi^{2}\right)$, a term depending on the renormalization scale $\mu$ and a $\tau_{Q}$ dependent piece $c\left(\tau_{Q}\right)$,

$$
C\left(\tau_{Q}\right)=\pi^{2}+c\left(\tau_{Q}\right)+\frac{33-2 N_{F}}{6} \ln \frac{\mu^{2}}{m_{H}^{2}} .
$$

The term $c\left(\tau_{Q}\right)$ can be reduced analytically to a twodimensional Feynman-parameter integral which has been performed numerically $[12,13]$. In the heavy-quark limit $\tau=m_{H}^{2} / 4 m_{Q}^{2} \ll 1$, the coefficient approaches the value $c\left(\tau_{Q}\right) \rightarrow \frac{11}{2}[6,7]$.

The (nonsingular) contributions from gluon radiation in $g g$ scattering, from $g q$ scattering, and $q \bar{q}$ annihilation depend on the renormalization scale $\mu$ and on the factorization scale $M$ of the parton densities [Figs. 1(b)-1(d)],

$$
\begin{aligned}
& \triangle \sigma_{g g}=\int_{\tau_{H}}^{1} d \tau \frac{d \mathcal{L}^{g g}}{d \tau} \frac{\alpha_{s}}{\pi} \sigma_{0}\left\{-z P_{g g}(z) \ln \frac{M^{2}}{\tau s}+d_{g g}\left(z, \tau_{Q}\right)+12\left[\left(\frac{\ln (1-z)}{1-z}\right)_{+}-z[2-z(1-z)] \ln (1-z)\right]\right\}, \\
& \triangle \sigma_{g q}=\int_{\tau_{H}}^{1} d \tau \sum_{q, \bar{q}} \frac{d \mathcal{L}^{g q}}{d \tau} \frac{\alpha_{s}}{\pi} \sigma_{0}\left\{\left[-\frac{1}{2} \ln \frac{M^{2}}{\tau s}+\ln (1-z)\right] z P_{g q}(z)+d_{g q}\left(z, \tau_{Q}\right)\right\} \\
& \triangle \sigma_{q \bar{q}}=\int_{\tau_{H}}^{1} d \tau \sum_{q} \frac{d \mathcal{L}^{q \bar{q}}}{d \tau} \frac{\alpha_{s}}{\pi} \sigma_{0} d_{q \bar{q}}\left(z, \tau_{Q}\right),
\end{aligned}
$$

with $z=\tau_{H} / \tau$. The renormalization scale enters through $\alpha_{s}\left(\mu^{2}\right)$ and $\sigma_{0}\left[\alpha_{s}\left(\mu^{2}\right)\right] . P_{g g}$ and $P_{g q}$ are the standard Altarelli-Parisi $g \rightarrow g$ and $q \rightarrow g$ splitting functions [14]. $F_{+}$denotes the usual + distribution such that $F(z)_{+}=$ $F(z)-\delta(z-1) \int_{0}^{1} d x F(x)$. The coefficients $d_{g g}, d_{g q}$, and $d_{q \bar{q}}$ can be reduced to one-dimensional integrals which have been evaluated numerically [13]. In the heavy-quark limit, the coefficients can be determined analytically [6, $7], d_{g g} \rightarrow-\frac{11}{2}(1-z)^{3}, d_{g q} \rightarrow-1+2 z-\frac{1}{3} z^{2}$, and $d_{q \bar{q}} \rightarrow$ $\frac{32}{27}(1-z)^{3}$.
The final results of our analysis are presented in Figs. 2 and 3 for LHC $(\sqrt{s}=16 \mathrm{TeV})$ and $\operatorname{SSC}(\sqrt{s}=40 \mathrm{TeV})$ energies. They are based on a top-quark mass of 140 $\mathrm{GeV}$ and, if not stated otherwise, on the DFLM (DemiozFerroni-Longo-Martinelli) parametrization [15] for the parton densities [16]. These parton densities have been transformed into the $\overline{\mathrm{MS}}$ scheme with a $\Lambda$ value $\Lambda \frac{(5)}{\mathrm{MS}}=$ $207 \mathrm{MeV}$ for $N_{F}=5$ quark flavors. The scale dependence of the QCD coupling $\alpha_{s}\left(\mu^{2}\right)_{\overline{\mathrm{MS}}}$ is used in next-toleading order, with the standard matching condition [17] 

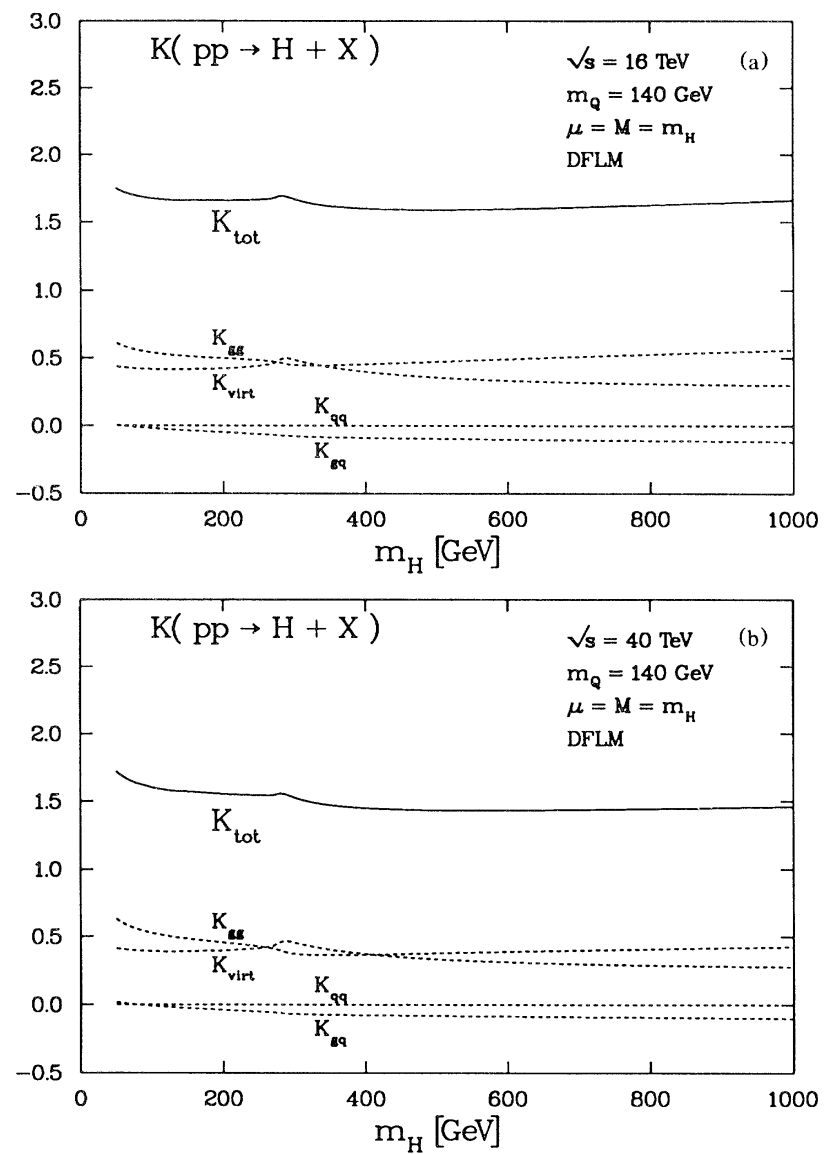

FIG. 2. $K$ factors of the QCD corrected cross sections $\sigma(p p \rightarrow H) ; K_{\text {virt }}$, regularized virtual corrections; $K_{A B}$, real corrections $(A, B=q, g) ; K_{\text {tot }}$, ratio of the QCD corrected cross section to the lowest order cross section. (a) LHC with $\sqrt{s}=16 \mathrm{TeV}$; (b) SSC with $\sqrt{s}=40 \mathrm{TeV}$.

at $\mu=m_{t}$. The cross section is sensitive to gluon and quark densities at $x$ values of order $10^{-3}-10^{-2}$ so that subtle nonlinear effects in the evolution at small $x$ need not be taken into account yet. More technical details will be described in a forthcoming publication.

We introduce $K$ factors in the standard way, $K_{\text {tot }}=$ $\sigma_{\mathrm{HO}} / \sigma_{\mathrm{LO}}$. The cross sections in next-to-leading order are normalized to the cross sections $\sigma_{\mathrm{LO}}$, evaluated for parton densities and $\alpha_{s}$ in leading order [18]. $K_{\text {virt }}$ accounts for the (regularized) virtual corrections; $K_{A B}$ for the real corrections, both defined in Eqs. (4)-(6) in the $\overline{\mathrm{MS}}$ scheme. The $K$ factors are shown for LHC and SSC energies in Fig. 2 as functions of the Higgs boson mass. For both the renormalization and the factorization scale $\mu=M=m_{H}$ has been chosen. Apparently $K_{\text {virt }}$ and $K_{g g}$ are of the same size and of the order of $50 \%$ while $K_{g q}$ and $K_{q \bar{q}}$ are quite small. Apart from the threshold region for Higgs decays into $t \bar{t}$ pairs, $K_{\text {tot }}$ is insensitive to the Higgs mass. The absolute magnitude of the correction is positive and large, increasing the cross section for
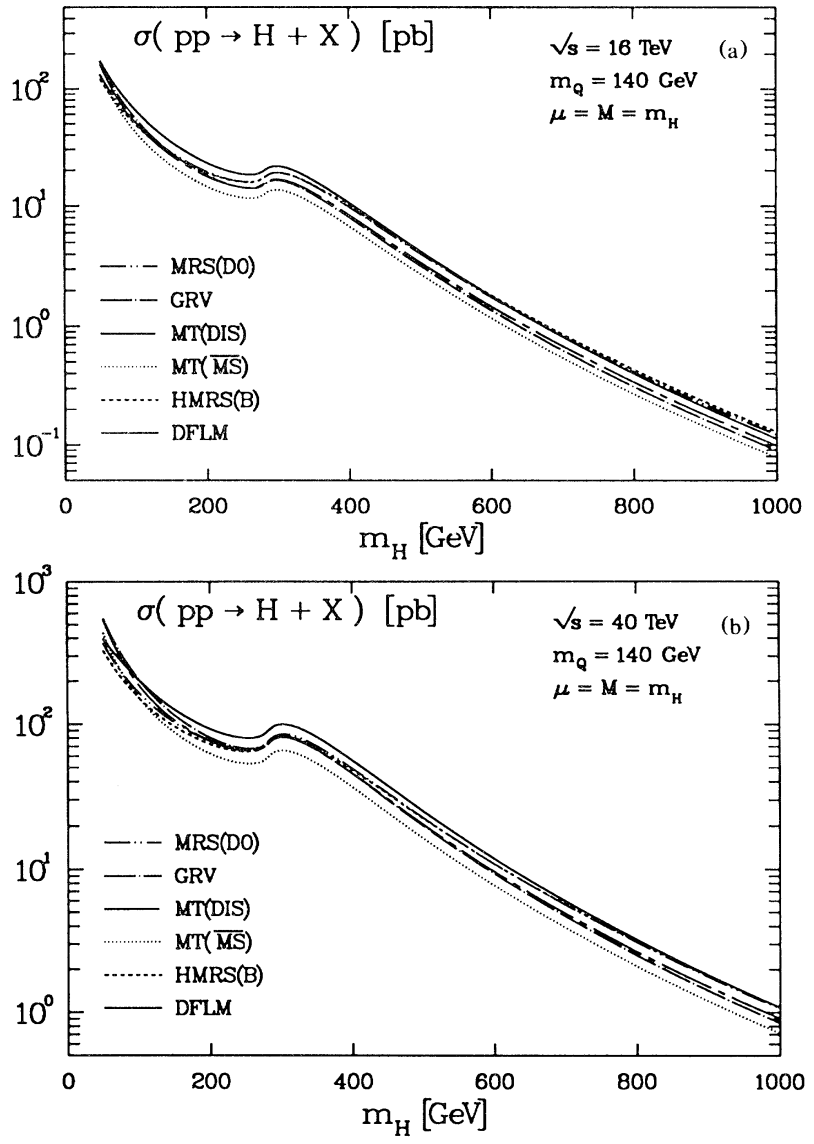

FIG. 3. The spread of the Higgs production cross section for various parametrizations $[15,19]$ of the parton densities. (a) LHC and (b) SSC for a top-quark mass of $140 \mathrm{GeV}$.

Higgs production at $p p$ colliders significantly by about a factor of 1.5 to 1.7. Comparing the exact numerical results with the analytic expressions in the heavy-quark limit, it turns out that these asymptotic solutions provide an excellent approximation even for Higgs boson masses above the top-quark decay threshold. (For Higgs masses below $\sim 700 \mathrm{GeV}$, the deviations of the QCD corrections from the asymptotic approximation are less than $15 \%$.)

The (unphysical) variation of the cross section with the renormalization and factorization scales is significantly reduced by including the next-to-leading corrections. While for $m_{H}=500 \mathrm{GeV}$ and $\sqrt{s}=16 \mathrm{TeV}$, for instance, the ratio $\sigma_{\mathrm{LO}}\left(\mu=\frac{1}{2} m_{H}\right) / \sigma_{\mathrm{LO}}\left(\mu=2 m_{H}\right)$ is found to be 1.63 (DFLM-LO [15] parametrization of the parton densities), this ratio drops to 1.32 in the next-toleading-order approximation. For $\sqrt{s}=40 \mathrm{TeV}$ this ratio falls from 1.42 down to 1.23. Besides the residual scale dependence, the main uncertainty in the prediction of the Higgs production cross section is due to the gluon density. Adopting a set of representative parton parametrizations $[15,19]$, we find a variation of about $40 \%$ between the maximum and minimum value of the 
cross section for Higgs boson mass values above $~ 100$ $\mathrm{GeV}$. This uncertainty will be reduced drastically in the near future when new data will become available from deep inelastic lepton-nucleon scattering experiments, in particular, at the DESY ep collider HERA.

Though the analysis has been carried out in detail for the production of Higgs bosons in the standard model, it is clear that the results can readily be transcribed, mutatis mutandis, to all other $C P$-even Higgs bosons in extended Higgs sectors. A well-known example is provided by the minimal supersymmetric extension of the standard model in which two Higgs doublets lead to two neutral $C P$-even particles $h$ and $H$ and a $C P$-odd particle $A$. Depending on the Higgs-fermion coupling, triangle $b$-quark loops may provide major contributions to the effective Higgs-boson-gluon-gluon couplings [20]. Since, apart from minor corrections, the $K$ factors are determined by the ratio of the Higgs boson to the quark-loop mass, the results in Fig. 2 can directly be reinterpreted for $h, H$ production after renormalizing the $m_{H}$ axis by the factor $m_{b} / m_{t}$ for $b$ loops. The cross section for the Higgs boson $A$ requires a careful reanalysis of the effective $C P$-odd vertex.

We have greatly benefited from discussions with $\mathrm{M}$. Chanowitz, S. Ellis, I. Hinchliffe, J. Kühn, Z. Kunszt, and J. van der Bij. D.G. thanks I. Hinchliffe for the warm hospitality extended to him at the Lawrence Berkeley Laboratory. This work was supported in part by the German Science Foundation DFG and the Bundesministerium für Forschung und Technologie. D.G. acknowledges the financial support of the Max-Kade Foundation (New York).

(a) Permanent address.

[1] P.W. Higgs, Phys. Lett. 12, 132 (1964); Phys. Rev. Lett. 13, 508 (1964); Phys. Rev. 145, 1156 (1966); F. Englert and R. Brout, Phys. Rev. Lett. 13, 321 (1964); G.S. Guralnik, C.R. Hagen, and T.W. Kibble, Phys. Rev. Lett. 13, 585 (1964).

[2] For recent reviews, see M.S. Chanowitz, Annu. Rev. Nucl. Part. Phys. 38, 323 (1988); G. Altarelli, Report No. CERN-TH 6092/91 (unpublished); J. Jersak, Jülich Report No. HLRZ 90-55 (unpublished); J.F. Gunion, H.E. Haber, G.L. Kane, and S. Dawson, The Higgs Hunter's Guide (Addison-Wesley, Reading, MA, 1990).

[3] L. Rolandi, in Proceedings of the Twenty-Sixth International Conference on High Energy Physics, Dallas, 1992 (to be published).

[4] See, e.g., D. Denegri, Report No. CERN 90-10, Vol. I (unpublished); SDC Design Report No. SSCL-SR-1215 (unpublished); Z. Kunszt, in Proceedings of the International Lepton-Photon Symposium and Europhysics Conference on High-Energy Physics, Geneva, 1991 (Zürich Report No. ETH-TH-91-28).

[5] H.M. Georgi, S.L. Glashow, M.E. Machazek, and D.V. Nanopoulos, Phys. Rev. Lett. 40, 692 (1978).
[6] A. Djouadi, M. Spira, and P.M. Zerwas, Phys. Lett. B 264, 441 (1991).

[7] S. Dawson, Nucl. Phys. B368, 283 (1991).

[8] I. Hinchliffe and S.F. Novaes, Phys. Rev. D 38, 3475 (1988); R.K. Ellis, I. Hinchliffe, M. Soldate, and J.J. van der Bij, Nucl. Phys. B297, 221 (1988); U. Baur and E.W.N. Glover, Nucl. Phys. B339, 38 (1990); R.P. Kauffman, Phys. Rev. D 44, 1415 (1991); 45, 1512 (1992); C. P. Yuan, Phys. Lett. B 283, 395 (1992); S. Dawson and R.P. Kauffman, Phys. Rev. Lett. 68, 2273 (1992).

[9] Beyond the mass range of $\sim 700 \mathrm{GeV}, W W$ fusion is the dominant production mechanism; see, e.g., R. Cahn and S. Dawson, Phys. Lett. 136B, 196 (1984); G. Kane, W. Repko, and W. Rolnick, Phys. Lett. 148B, 367 (1984). Radiative QCD corrections have recently been shown to be small for this mechanism; T. Han, G. Valencia, and S. Willenbrock, Phys. Rev. Lett. 69, 3274 (1992).

[10] E. Braaten and J.P. Leveille, Phys. Rev. D 22, 715 (1980)

[11] G. Altarelli, R.K. Ellis, and G. Martinelli, Nucl. Phys. B157, 461 (1979); W. Furmanski and R. Petronzio, Z. Phys. C 11, 293 (1982).

[12] The analytic functions which have been integrated numerically, include singularities like $\ln (x+i \epsilon)$ and $1 /(x+$ $i \epsilon)$. The integrals have been solved for small positive $\epsilon$ values varied from $10^{-2}$ downward. At $10^{-3}$ a plateau was reached which extends down to $10^{-4}$. Below this value, the numerical integration became unstable.

[13] Details will be given in an extended version of this paper; see also M. Spira, Ph.D. thesis, Rheinisch-Westfalische Technische Hochschule Aachen, October 1992

[14] G. Altarelli and G. Parisi, Nucl. Phys. B126, 298 (1977).

[15] M. Demioz, F. Ferroni, E. Longo, and G. Martinelli, Z. Phys. C 39, 21 (1988).

[16] It is well known that such DIS based gluon densities suffer from uncertainties beyond the leading order in that they enter the analysis only as a higher order correction. These uncertainties could eventually be removed by exploiting gluon initiated processes like direct photon production or heavy-quark production which allow for a direct physical definition of the gluon distribution; see, e.g., P. Nason, S. Dawson, and R.K. Ellis, Nucl. Phys. B303, 607 (1988).

[17] W.J. Marciano, Phys. Rev. D 29, 580 (1984); W. Bernreuther, Ann. Phys. (N.Y.) 151, 127 (1983).

[18] Residual uncertainties beyond the leading order in these $K$ factors, as for all other gluon initiated processes, are caused by extracting the gluon densities in the DIS scheme, as emphasized in the preceding reference.

[19] P.N. Harriman, A.D. Martin, W. J. Stirling, and R. G. Roberts, Phys. Rev. D 42, 798 (1990); J. Morfin and Wu-Ki Tung, Z. Phys. C 52, 13 (1991); A. Martin, R. Roberts, and J. Stirling, Durham Report No. DTP-92-16 (to be published); M. Glück, E. Reya, and A. Vogt, Z. Phys. 48, 471 (1990); C 53, 127 (1992).

[20] Z. Kunszt and F. Zwirner, Zürich Report No. ETH-TH91-7 (to be published); V. Barger, M. Berger, S. Stange, and R. Phillips, Phys. Rev. D 45, 4128 (1992); H. Baer, M. Bisset, C. Kao, and X. Tata, Phys. Rev. D 46, 1067 (1992); J. Gunion and L. Orr, Phys. Rev. D 46, 2052 (1992). 